Original Article

\title{
SPA0355 prevents ovariectomy-induced bone loss in mice
}

\author{
Sang Hoon Kim ${ }^{1, \#}$, Zhongkai Zhang ${ }^{2, \#}$, Young Jae Moon ${ }^{3, \#}$, Il Woon Park ${ }^{4}$, Yong Gon Cho ${ }^{5}$, Raok Jeon ${ }^{6, *}$, and \\ Byung-Hyun Park ${ }^{3, *}$
}

Departments of ${ }^{1}$ Surgery, ${ }^{2}$ Orthopaedic Surgery, ${ }^{3}$ Biochemistry, and ${ }^{5}$ Laboratory Medicine, Chonbuk National University Medical School, Jeonju 54896, Korea ${ }^{4}$ Department of Cognitive Science, Case Western Reserve University, Cleveland, OH 44106, USA, ${ }^{6}$ College of Pharmacy, Sookmyung Women's University, Seoul 04310, Korea

\section{ARTICLE INFO}

Received July 24,2018

Revised November 6, 2018

Accepted November 15, 2018

\section{*Correspondence}

Raok Jeon

E-mail: rjeon@sm.ac.kr

Byung-Hyun Park

E-mail: bhpark@jbnu.ac.kr

\section{Key Words}

Osteoblastogenesis

Osteoclastogenesis

Osteoporosis

Ovariectomy

SPA0355

\#These authors contributed equally to this work.

\begin{abstract}
Estrogen withdrawal in post-menopausal women leads to overactivation of osteoclasts, which contributes to the development of osteoporosis. Inflammatory cytokines are known as one of mechanisms of osteoclast activation after estrogen deficiency. SPA0355 is a thiourea derivative that has been investigated for its antioxidant and anti-inflammatory activities. However, its efficacy in bone resorption has not been previously investigated. The aim of this study was to investigate the impact of SPA0355 on the development of osteoporosis and to explore its mode of action. In vitro experiments showed that SPA0355 inhibited receptor activator of NF- $\mathrm{KB}$ ligand (RANKL)-induced osteoclastogenesis in primary bone marrow-derived macrophages. This effect appears to be independent of estrogen receptor activation as ICI 180,782 failed to abrogate its effects on osteoclasts. Further signaling studies revealed that SPA0355 suppressed activation of the MAPKs, Akt, and NF-אB pathways. SPA0355 also increased osteoblastic differentiation, as evidenced by its effects on alkaline phosphatase activity and mineralization nodule formation. Intraperitoneal administration of SPA0355 to ovariectomized mice prevented bone loss, as verified by threedimensional images and bone morphometric parameters derived from $\mu \mathrm{CT}$ analysis. Noticeably, SPA0355 did not show hepatotoxicity and nephrotoxicity and also had little effect on hematological parameters. Taken together, the results indicate that SPA0355 may protect against bone loss in ovariectomized mice by stimulation of osteoblast differentiation and by inhibition of osteoclast resorption. Therefore, SPA0355 is a safe and potential candidate for management of postmenopausal osteoporosis.
\end{abstract}

\section{INTRODUCTION}

An imbalance between osteoblastic bone formation and osteoclastic bone resorption reduces bone mineral density and increases risk of fracture. In most cases, an increase in the ratio of osteoclast-to-osteoblast is due to estrogen deficiency during menopause [1]. In estrogen deficiency, receptor activator of nuclear factor- $\mathrm{KB}$ ligand (RANKL) is secreted from osteocytes and $B$ cells [2]. Binding of RANKL to RANK on osteoclast progenitor cells leads to the recruitment of TNF receptor-associated factors (TRAFs) and subsequent activation of mitogen-activated protein kinases (MAPKs) and nuclear factor- $\kappa \mathrm{B}(\mathrm{NF}-\kappa \mathrm{B})$ pathways [35]. These signaling pathways further induce nuclear factor of activated T cells $\mathrm{cl}$ (NFATc1), a master regulator of osteoclast differentiation, and target the expression of genes such as matrix metallopeptidase $9(\mathrm{Mmp} 9)$, osteoclast-associated receptor $(\mathrm{Os}$ car), tartrate-resistant acid phosphatase 5 (Acp5), integrin beta 3 (Itgb3), and cathepsin K (Ctsk) [6]. Taken together, these findings suggest that the RANKL-RANK-TRAFs-NFATc1 axis is essential for the formation of mature osteoclasts after menopause.

Previously, we synthesized SPA0355 (1-methyl-3-[4-(2-phenoxazin-10-ylethoxy)phenyl]thiourea) and reported its anti-inflam- (i) (\$) This is an Open Access article distributed under the terms of the Creative Commons Attribution Non-Commercial License, which permits unrestricted non-commercial use, distribution, and reproduction in any medium, provided the original work is properly cited. Copyright @ Korean J Physiol Pharmacol, pISSN 1226-4512, elSSN 2093-3827
Author contributions: S.H.K., Z.Z., Y.J.M., Y.G.C., and I.W.P. performed experiments and analyzed the data. R.J. and B.H.P. designed the experiments, interpreted the data, and wrote the manuscript. All authors reviewed the manuscript. 
matory effects in animal experimental models of rheumatoid arthritis [7], type 1 diabetes [8], hepatic ischemia-reperfusion injury [9], and allergic airway inflammation [10]. Biological actions of SPA0355 were related to the suppression of NF- $\kappa$ B and Janus kinase (JAK)/signal transducer and activator of transcription (STAT) pathways. Based on these observations, we speculated a possible beneficial effect of SPA0355 on ovariectomy (OVX)induced bone loss in mice. Our results demonstrate that SPA0355 has preventive effects for osteoporosis by counteracting osteoclast-mediated bone loss and by stimulating osteoblast formation.

\section{METHODS}

\section{Preparation of SPA0355}

SPA0355 was prepared as previously described [7].

\section{Animals and experimental design}

Pathogen-free 10-week-old C57BL/6 female mice were purchased from Orient Bio (Seoul, Korea). Mice were housed in a laminar flow cabinet with a $12 \mathrm{~h}$ light/dark cycle and maintained on standard laboratory chow ad libitum. Mice were anesthetized with ketamine $(100 \mathrm{mg} / \mathrm{kg})$ and xylazine $(10 \mathrm{mg} / \mathrm{kg})$ by intraperitoneal injection and subjected to either a sham operation or bilateral OVX. Mice were divided into four groups ( $n=5$ per group): sham, vehicle (OVX+100 $\mu$ l corn oil), and one of two doses of SPA0355 (OVX+10 or $50 \mathrm{mg} / \mathrm{kg}$ SPA0355 in $100 \mu \mathrm{l}$ corn oil). The administration of vehicle or SPA0355 was started from day three after surgery and continued three times per week for five weeks. At the end of the study, femurs were fixed in $4 \%$ paraformaldehyde for $\mu \mathrm{CT}$ analyses. In toxicity study, SPA0355 was administered intraperitoneally at the dose of $50 \mathrm{mg} / \mathrm{kg} /$ day for seven consecutive days. This protocol was approved by the Institutional Animal Care and Use Committee at Chonbuk National University (permit number: CBNU 2015-87) and all work was performed in accordance with the National Institutes of Health guide for the care and use of Laboratory animals (NIH Publications No. 8023, revised 1978).

\section{Dual energy $x$-ray absorptiometry (DEXA) and micro- computed tomography $(\mu \mathrm{CT})$}

Five weeks after surgery, mice were anesthetized through ip injection of ketamine/xylazine, and their femur bone mineral densities (BMDs) were measured using a Lunar PIXImus densitometer (GE Lunar PIXImus Corporation Headquarters, Madison, WI, USA). Mice were then sacrificed, and structural changes in the femur were analyzed by SKYSCAN 1076 Micro-CT (Skyscan, Kontich, Belgium). The fixed femurs were scanned and reconstructed into three-dimensional images using NRECON software (version
1.6.1.5) and CT analyzer (version 1.10.0.1), both from SkyScan. Bone volume (BV), total volume (TV), percent bone volume (BV/ $\mathrm{TV})$, bone surface (BS), bone surface density (BS/TV), trabecular thickness (Tb.Th), and trabecular separation (Tb.Sp) were measured using the resident reconstruction program (Skyscan). A global algorithm was applied at a constant threshold for all specimens. The threshold was the intensity (gray value) that corresponded to $\sim 50 \%$ of the average intensity of intact cortical bone. Trabecular lesions or metaphyseal cortical bone were analyzed in the distal metaphysis extending proximally $2.5 \mathrm{~mm}$ from the end of the growth plate.

\section{Histological study}

Fixed liver and kidney tissues were embedded in paraffin. Tissue sections $(5 \mu \mathrm{m})$ were stained with hematoxylin and eosin (H\&E) for light microscopy.

\section{Hematological and biochemical assays}

The plasma levels of hemoglobin $(\mathrm{Hb})$, hematocrit $(\mathrm{Hct})$, and the number of white blood cell (WBC) and platelet (PLT) were measured using automated hematology analyzer (XN-2000, Sysmex Co., Kobe, Japan). The serum levels of alanine aminotransferase (AST), aspartate aminotransferase (AST), blood urea nitrogen (BUN) and creatinine were measured using automated chemistry analyzer (ADVIA 2400, Siemens Healthcare Diagnostics Inc., Tarrytown, NY, USA).

\section{Osteoclast differentiation}

Bone marrow-derived macrophages (BMMs) were prepared as previously described [11]. BMMs were seeded at $2 \times 10^{6}$ cells/ well in 6-well plates and cultured with macrophage colonystimulating factor (M-CSF; Peprotech, London, United Kingdom; $30 \mathrm{ng} / \mathrm{ml}$ ). After incubation for two days, cells were cultured in the presence of $10 \mathrm{ng} / \mathrm{ml} \mathrm{M-CSF}$ and $30 \mathrm{ng} / \mathrm{ml}$ soluble RANKL (sRANKL, Peprotech). To detect formation and calculate the number of osteoclasts, BMMs were seeded at $5 \times 10^{4}$ cells/well in 48-well plates and cultured with M-CSF $(10 \mathrm{ng} / \mathrm{ml})$ and sRANKL (30 ng/ml) for four days. Osteoclasts were visualized by staining for tartrate-resistant acid phosphatase (TRAP) activity (SigmaAldrich, St. Louis, MO, USA). TRAP-positive cells were counted as osteoclasts.

\section{Alizarin red S staining}

MC3T3-E1 cells $\left(5 \times 10^{4}\right.$ cells/well) were cultured on 24-well plates and incubated in the differentiation medium $(50 \mu \mathrm{g} / \mathrm{ml}$ ascorbic acid and $10 \mathrm{mM} \beta$-glycerophosphate) for 21 days. Then cells were fixed in 70\% ice-cold ethanol and stained with 2\% Alizarin red S. After washing with PBS, 10\% cetylpyridinium chlo- 
ride solutions was added to dissolve the calcium precipitates and absorbance was measured at $560 \mathrm{~nm}$ with a microplate reader.

\section{Alkaline phosphate (ALP) staining and activity}

MC3T3-E1 cells $\left(5 \times 10^{4}\right.$ cells/well) were cultured in 24-well plates and then incubated in the differentiation medium. On day 6, cells were fixed with $70 \%$ ice-cold methanol and stained with ALP staining solution (Sigma-Aldrich). For ALP enzymatic activity, cells were lysed at Day 6 with cell lysis buffer, and cell lysates were incubated with p-nitrophenyl phosphate (p-NPP) (Abcam, Cambridge, MA, USA). The color change of p-NPP to p-nitrophenol was measured at a wavelength of $405 \mathrm{~nm}$.

\section{RNA isolation and real-time quantitative RT-PCR (qPCR)}

Total RNA was extracted from differentiated osteoclasts using an RNA Iso kit (TaKaRa, Tokyo, Japan). cDNA was prepared by reverse transcribing $1 \mu \mathrm{g}$ of RNA with the first-strand cDNA synthesis kit (Applied Biosystems, Foster City, CA, USA). Primer sequences of genes used for quantification of mRNA by real-time PCR appear in Table 1. qPCR was performed using an ABI Prism 7900HT Sequence Detection System (Applied Biosystems).

\section{Western blotting}

Cell lysates $(20 \mu \mathrm{g})$ were separated by 10\% SDS-PAGE and transferred to PVDF membranes. After blocking with 5\% nonfat dry milk, the membrane was probed with primary antibodies against p38, p-p38, p-AKT, AKT, p-ERK, ERK, JNK, p-JNK, p65, $\mathrm{p}$-IкB $\mathrm{B}, \mathrm{I}_{\kappa} \mathrm{B} \alpha$ (Cell Signaling, Beverly, MA, USA), or HSP90 (Enzo Life Sciences, Farmingdale, NY, USA). Membrane was incubated with goat anti-rabbit horseradish peroxidase-conjugated

Table 1. Sequences and accession numbers for primers (forward, FOR; reverse, REV) used in real-time RT-PCR

\begin{tabular}{cll}
\hline Gene & \multicolumn{1}{c}{ Sequences for primers } & Accession No. \\
\hline Mmp99 & FOR: TAGCTACCTCGAGGGCTTCC & NM_013599 \\
& REV: GTGGGACACATAGTGGGAGG & \\
Oscar & FOR: TCGCTGATACTCCAGCTGTC & NM_175632 \\
& REV: ATCCCAGGAGTCACAACTGC & \\
Acp5 & FOR: CGTCTCTGCACAGATTGCAT & NM_007388 \\
& REV: GAGTTGCCACACAGCATCAC & \\
Itgb3 & FOR: GGCGTTGTTGTTGGAGAGTC & NM_016780 \\
& REV: CTTCAGGTTACATCGGGGTGA \\
Ctsk & FOR: GCAGGATGTGGGTGTTCAAG T NM_007802 \\
& REV: TCCAGCATTTCCTCCGGAG \\
Nfatc1 & FOR: AATTAGGAGTGGGGGATCGT & NM_001164109 \\
& REV: ATCCAACCCAACTCGCCT & \\
Actb & FOR: ATGGAGGGGAATACAGCCC & NM_007393 \\
& REV: TTCTTTGCAGCTCCTTCGTT & \\
\hline
\end{tabular}

IgG (Santa Cruz Biotechnology, Santa Cruz, CA, USA) for $1 \mathrm{~h}$. The proteins of interest on immunoblots were detected by using Las-4000 imager (GE Healthcare Life Science, Pittsburgh, PA, USA).

\section{Luciferase}

Transient transfections were performed using Lipofectamine 3000 (Invitrogen, Carlsbad, CA, USA) according to the manufacturer's instructions. Briefly, BMMs were transfected with plasmids encoding the NF- $\mathrm{KB}$-luc reporter and the renilla luciferase reporter (pRL-TK-luc). The day following transduction, cells were treated with $30 \mathrm{ng} / \mathrm{ml} \mathrm{sRANKL}$, either with or without a $2 \mathrm{~h}$ pretreatment with $10 \mu \mathrm{M}$ SPA0355. After $30 \mathrm{~min}$, cells were harvested in reporter lysis buffer (Promega, Madison, WI, USA). Luciferase activity was determined in whole cell lysates using the Promega luciferase assay kit and is expressed relative to the renilla signal.

\section{Statistical analysis}

All results are expressed as the mean \pm SEM. Statistical comparisons were made using one-way ANOVA followed by Fisher's post hoc analysis using GraphPad Prism 5.02 (San Diego, CA, USA). $\mathrm{p}<0.05$ was considered statistically significant.

\section{RESULTS}

\section{SPA0355 prevents OVX-induced bone loss}

To determine whether SPA0355 could prevent bone loss induced by estrogen deficiency, mice were either sham-operated or ovariectomized with or without SPA0355 treatment. Five weeks after OVX, body weights were significantly increased compared to the sham operated mice (Fig. 1A). SPA0355 treatment at either dose $(10$ or $50 \mathrm{mg} / \mathrm{kg})$ did not affect body weight significantly when compared to the untreated mice. In contrast to the body weight increase, the uterine weights decreased in the OVX mice when compared to the sham group (Fig. 1B). Again, SPA0355 treatment did not alter the uterine weight of OVX mice.

Next, we compared changes in bone mass. Ovariectomy caused a marked decrease of BMD in mice; however, treatment of SPA0355 (10 or $50 \mathrm{mg} / \mathrm{kg})$ led to a significant increase of BMD compared to OVX mice (Fig. 2A). Consistently, $\mu \mathrm{CT}$ analyses also revealed bone loss in OVX mice: BV, BV/TV, BS, BS/TV, and $\mathrm{Tb} . T h$ were decreased, while Tb.Sp was increased in femurs compared to those of the sham group (Figs. 2B and C). However, the changes of these parameters were significantly attenuated in SPA0355-treated mice. 


\section{SPA0355 does not cause tissue damage}

To investigate the potential side-effect of SPA0355, we administered SPA0355 daily for seven days. As shown in Table 2, SPA0355 at dose $50 \mathrm{mg} / \mathrm{kg}$ did not alter hematological parameters such as hemoglobin, hematocrit, white blood cell, and platelet. Plasma
A

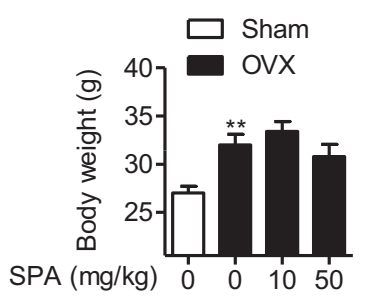

B

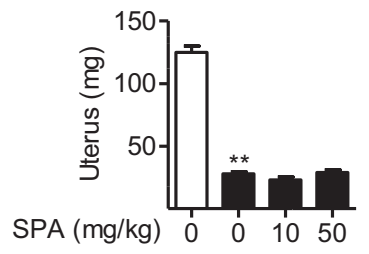

Fig. 1. Effects of SPA0355 on body weight and uterine weight of OVX mice. OVX mice were treated with vehicle or SPA0355 (10 or 50 $\mathrm{mg} / \mathrm{kg}$, i.p.) for five weeks. At the end of the study, body weight (A) and uterine weight $(B)$ were measured. Values are the mean $\pm \operatorname{SEM}(n=5)$. ${ }^{* *} \mathrm{p}<0.01$ vs. sham-operated mice. SPA, SPA0355. levels of AST, ALT, BUN, and creatinine (Table 2) and histological observations of liver and kidney (Fig. 3) showed no specific tissue damage by SPA0355 treatment when compared to the control mice.

\section{SPA0355 inhibits osteoclast differentiation}

To determine whether SPA0355 inhibits osteoclast precursors and their differentiation into osteoclasts, the effect of SPA0355 (10 and $20 \mu \mathrm{M}$ ) was examined for RANKL-induced osteoclast formation in BMMs. SPA0355 at these concentrations was not toxic to BMMs during the four-day differentiation period (Fig. 4A). BMMs differentiated into osteoclasts in the presence of MCSF and sRANKL. Treatment of SPA0355 decreased the formation of TRAP-positive multinuclear cell formation (Fig. 4B). In the presence of $20 \mu \mathrm{M}$ SPA0355, there was almost complete inhibition of osteoclastogenesis. We examined whether or not the inhibitory effect of SPA0355 on mature osteoclast formation is mediated via estrogen receptor. Treatment of BMMs with ICI 182,780, an estrogen receptor blocker, did not abrogate SPA0355 effect on osteoclast formation, indicating that SPA0355 action on
A

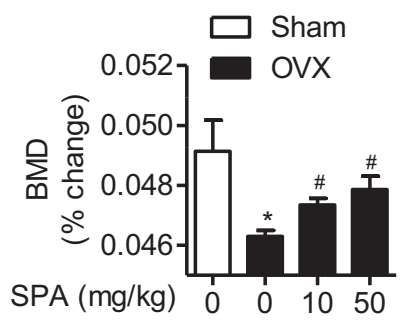

C
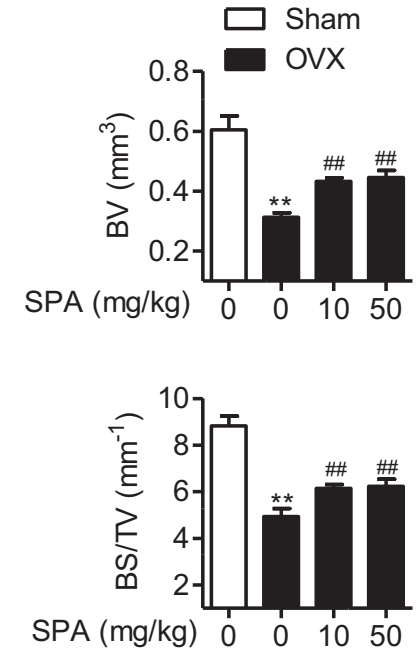

B

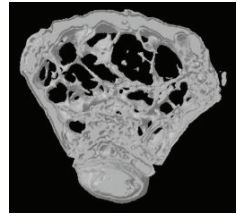

Sham

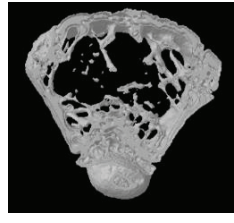

0

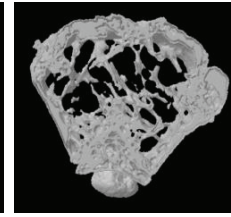

$10 \mathrm{mg} / \mathrm{kg}$

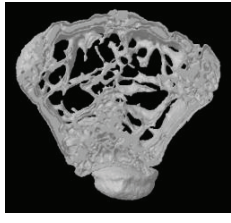

$50 \mathrm{mg} / \mathrm{kg}$
OVX+SPA0355
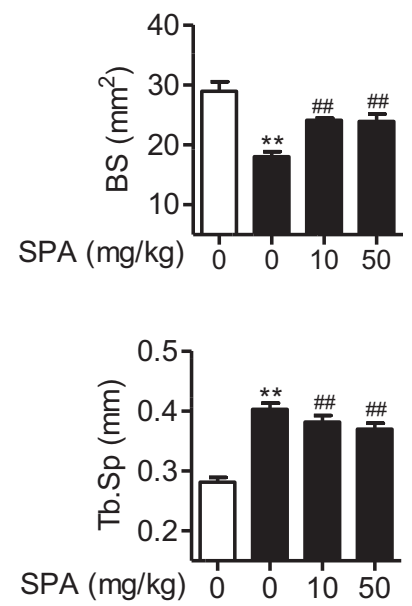

Fig. 2. Preventive effects of SPA0355 on bone loss in OVX mice. (A) Bone marrow density (BMD) was measured using DEXA and expressed as percent change from baseline values $(n=5)$. (B) Representative reconstructed $\mu C T$ images of femur. (C) Bone morphometric parameters (BV, BV/TV, BS, BS/ TV, Tb.Th, and Tb.Sp) were determined ( $n=5)$. Values are the mean \pm SEM. ${ }^{*} p<0.05$ and ${ }^{* *} p<0.01$ vs. sham-operated mice; ${ }^{*} p<0.05$ and ${ }^{\# \#} p<0.01$ vs. OVX mice. BV, bone volume; TV, total volume; BS, bone surface; Tb.Th, trabecular thickness; Tb.Sp, trabecular separation. 
Table 2. Effects of intraperitoneal administration of SPA0355 $(50 \mathrm{mg} / \mathrm{kg})$ on hematological and biochemical parameters in mice

\begin{tabular}{lcccccccc}
\hline & $\mathrm{Hb}(\mathrm{g} / \mathrm{dl})$ & $\mathrm{Hct}(\%)$ & WBC $\left(\times 10^{3} / \mu \mathrm{l}\right)$ & $\mathrm{PLT}\left(\times 10^{6} / \mu \mathrm{l}\right)$ & AST $(\mathrm{IU} / \mathrm{l})$ & ALT $(\mathrm{IU} / \mathrm{l})$ & $\mathrm{BUN}(\mathrm{mg} / \mathrm{dl})$ & $\mathrm{Cr}(\mathrm{mg} / \mathrm{dl})$ \\
\hline Vehicle & $12.7 \pm 0.2$ & $51 \pm 1$ & $3.8 \pm 0.6$ & $667 \pm 34$ & $57 \pm 2$ & $27 \pm 2$ & $22 \pm 1$ & $0.2 \pm 0.01$ \\
SPA0355 & $12.9 \pm 0.2$ & $52 \pm 1$ & $3.9 \pm 0.6$ & $699 \pm 12$ & $55 \pm 2$ & $27 \pm 1$ & $19 \pm 1$ & $0.2 \pm 0.01$ \\
\hline
\end{tabular}

Values are the mean \pm SEM. Hg, hemoglobin; Hct, hematocrit; WBC, white blood cell; PLT, platelet; AST, aspartate aminotransferase; ALT, alanine aminotransferase; $\mathrm{BUN}$, blood urea nitrogen; $\mathrm{Cr}$, creatinine.
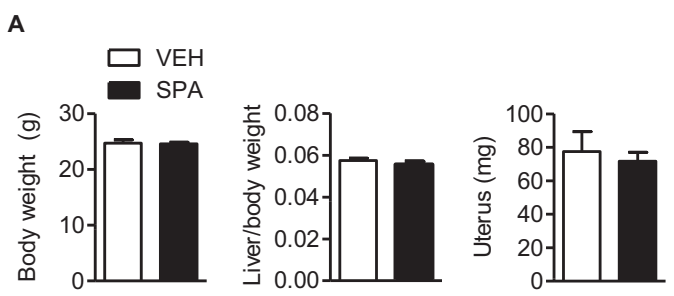

B

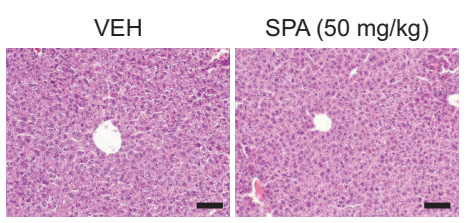

Fig. 3. Acute and subacute toxicity of SPA0355 on liver and kidney. SPA0355 ( $50 \mathrm{mg} / \mathrm{kg}$ body weight in $100 \mu \mathrm{l}$ of corn

C

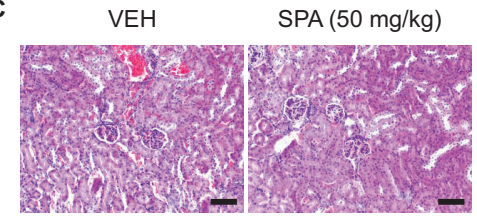
oil) was intraperitoneally injected daily for seven days. (A) Body weight, liver weight per body weight, and uterus weight were determined $(n=6)$. Tissue sections of liver (B) and kidney (C) were prepared and stained by $\mathrm{H} \& \mathrm{E}$. Bars $=250$ $\mu \mathrm{m}$.

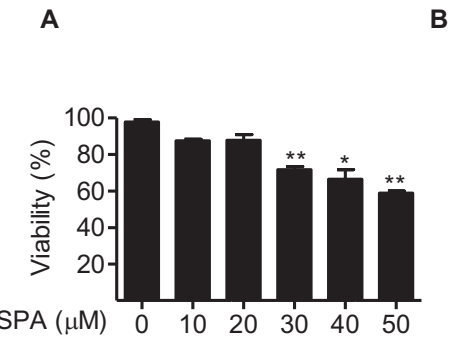

B
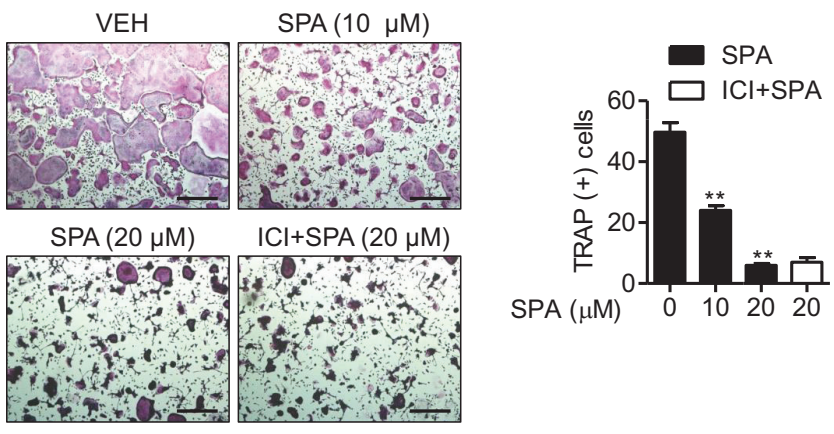

C
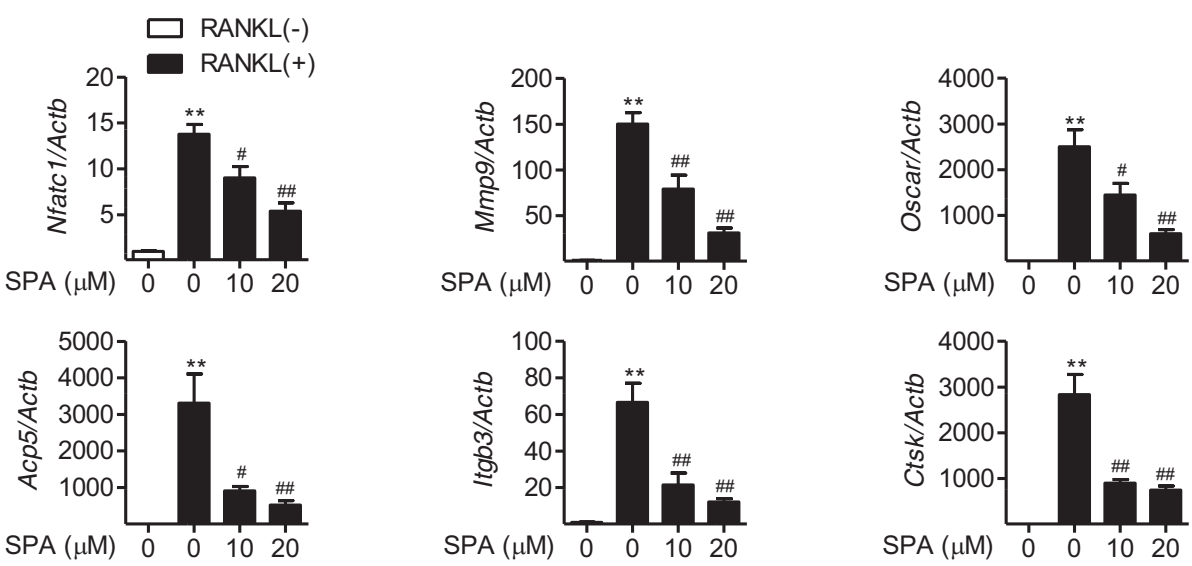

Fig. 4. Effects of SPA0355 on RANKL-induced osteoclast differentiation. (A) BMMs $\left(1 \times 10^{4}\right)$ were treated with the indicated concentrations of SPA0355 for four days, and cell viability was determined by MTT assay $(n=5)$. (B) BMMs were cultured for four days with M-CSF (10 ng/ml) and sRANKL $(30 \mathrm{ng} / \mathrm{ml})$ in the presence or absence of 10 and $20 \mu \mathrm{M}$ SPA0355. For ICl 182,780 treatment, $100 \mathrm{nM}$ compound was used $2 \mathrm{~h}$ prior to SPA0355 treatment. Cells were fixed with $3.7 \%$ formalin, permeabilized with $0.1 \%$ Triton X-100, and stained with TRAP solution. TRAP-positive multinucleate cells with three or more nuclei were scored as osteoclasts $(n=4)$. ICI, ICl 182,780. (C) The mRNA expression of osteoclastogenesis-related genes was analyzed by real-time RT-PCR ( $n=10-12)$. Values are the mean \pm SEM. ${ }^{*} p<0.05$ and ${ }^{* *} p<0.01$ vs. untreated control. ${ }^{*} p<0.05$ and ${ }^{\# \#} p<0.01$ vs. RANKL+VEH. 
A

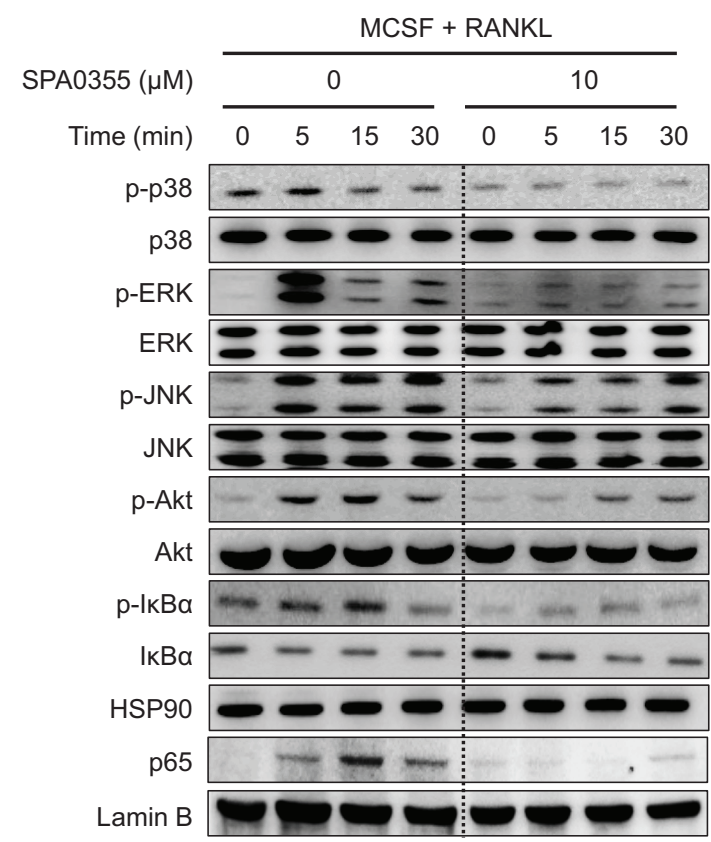

B

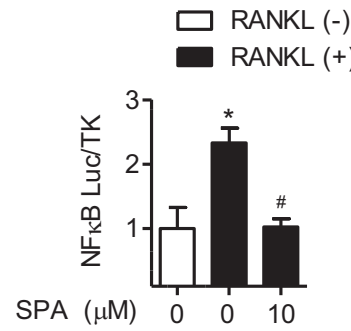

Fig. 5. Regulation of RANKL-activated signaling pathways by SPA0355. (A) BMMs were treated with M-CSF (10 ng/ml) and sRANKL (30 ng/ml) with or without $10 \mu \mathrm{M}$ SPA0355 for the indicated periods. Cell lysates were prepared, and the changes of MAPKs, Akt, and NF- $\mathrm{k} B$ were evaluated by western blotting. HSP90 and lamin B were used as loading controls for cytosolic and nuclear protein, respectively. (B) BMMs were transiently transfected with an NfkB luciferase construct and then treated with $30 \mathrm{ng} / \mathrm{ml}$ RANKL in the presence or absence of $10 \mu \mathrm{M}$ SPA0355. After $24 \mathrm{~h}$, cells were harvested in reporter lysis buffer, and luciferase activity in cell lysates was assayed $(n=4)$. Values are the mean $\pm S E M$. ${ }^{*} p<0.05$ vs. untreated control. \# $\mathrm{p}<0.05$ vs. RANKL+VEH.

the osteoclastogenesis is estrogen receptor-independent. We next examined the effect of SPA0355 on the expression of osteoclast marker genes. As shown in Fig. 4C, SPA0355 considerably decreased RANKL-induced mRNA levels of Mmp9, Oscar, Acp5, Itgb3, and Ctsk. SPA0355 also reduced RANKL-induced expression of $\mathrm{Nfatcl}$, which has been reported to be a crucial regulator of osteoclastogenesis [6].

\section{SPA0355 inhibits MAPKs, Akt, and NF-kB pathways in BMMs}

We examined the effect of SPA0355 on RANKL-induced signaling pathways in BMMs. RANKL rapidly induced the phosphorylation of p38, ERK, JNK, and Akt, and SPA0355 treatment inhibited all of these pathways (Fig. 5A). We also investigated whether SPA0355 could suppress the RANKL-induced NF- $\kappa$ B signaling pathway. SPA0355 inhibited RANKL-induced $I_{\kappa} B \alpha$ phosphorylation, NF- $\mathrm{B}$ nuclear translocation, and NF- $\kappa \mathrm{B}$ luciferase reporter activity (Figs. $5 \mathrm{~A}$ and $\mathrm{B}$ ). From these results, we could confirm that SPA0355 suppresses osteoclastogenesis by inhibiting RANKL-induced MAPKs, Akt, and NF- $\kappa$ B pathways.

\section{SPA0355 stimulates osteoblastogenesis}

Because the balance between bone formation by osteoblasts
A

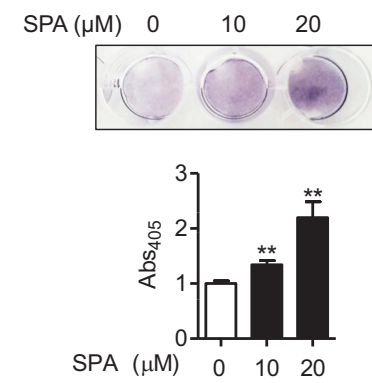

B

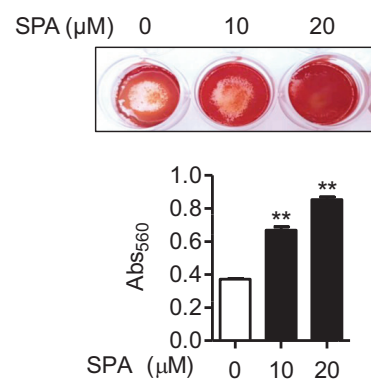

Fig. 6. Stimulation of osteoblast differentiation by SPA0355 in MC3T3-E1 cells. (A) MC3T3-E1 cells $\left(5 \times 10^{4}\right)$ were treated with SPA0355 in differentiation medium for seven days. Cells were fixed and stained with ALP staining. ALP activity is expressed as p-nitrophenol released $(n=3)$. (B) After 21 days of differentiation, calcium deposits for matrix mineralization were measured by Alizarin red $\mathrm{S}$ staining. The Alizarin Red dye was extracted using cetylpyridinium chloride $(n=3)$. Values are the mean \pm SEM. ${ }^{* *} p<0.01$ vs. vehicle.

and bone resorption by osteoclasts maintains adequate bone mass [1], we next questioned if SPA0355 has an osteogenic activity. We assessed the staining and activity of ALP, an early differentiation marker, in MC3T3-E1 cells. As shown in Fig. 6A, ALP staining and activity were significantly increased by SPA0355 at 10 and 20 


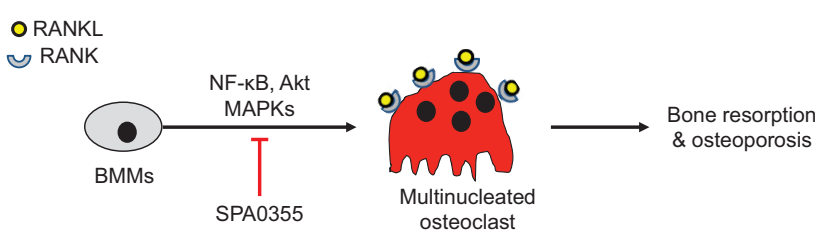

Fig. 7. Proposed summary.

$\mu \mathrm{M}$ concentrations. We also performed Alizarin red S staining to measure mineralization of the extracellular matrix as a marker for osteoblastic maturation. Treatment with SPA0355 significantly promoted bone nodule formation in a dose-dependent manner (Fig. 6B), indicating that SPA0355 acts on both initial and terminal steps of osteoblast differentiation.

\section{DISCUSSION}

The aims of this study were to elucidate the effects of SPA0355 on osteoclastogenesis and osteoblastogenesis in vitro and on OVX-induced bone loss in vivo. A previous study in our laboratory demonstrated that SPA 0355 potently inhibits bone resorption in ankle joints of collagen-induced arthritic mice [7]. In order to examine the in vivo effects of SPA0355 treatment on bone turnover, we used an OVX-induced osteoporosis model. In agreement with earlier reports $[12,13]$, OVX resulted in a significant increase of body weight with concomitant decrease of uterine weight, indicating the success of the OVX. SPA0355 treatment did not have significant effects on body weight or uterine weight when compared to the control. Given that estrogen is the main hormone produced by the ovary and induces uterine growth [14] and that ICI 182,780 does not affect the action of SPA0355 on osteoclast formation, the osteo-protective effects of SPA0355 on an OVXinduced osteoporosis model do not depend on estrogen signaling.

Estrogen deficiency also accelerates osteoclast activity by indirect mechanisms. Estrogen withdrawal induces secretion of cytokines such as TNF- $\alpha$, IL-6, IL-1, RANKL, and M-CSF from $\mathrm{T}$ cells, bone marrow stromal cells, osteoblasts, osteocytes, B cells. The cytokines promotes proliferation and differentiation of osteoclast precursors to mature osteoclast [2,15]. Especially, upon activation of RANK through binding of RANKL, the MAPKs, Akt, and NF- $\kappa \mathrm{B}$ pathways are activated in osteoclasts [3-5]. A number of small molecules and natural products have proven to inhibit osteoclastogenesis and bone loss by inhibiting these pathways both upstream and downstream of RANKL signaling [16,17]. Likewise, SPA0355 also inhibited MAPKs, Akt, and NF- $\mathrm{B}$ pathways, which led to decreased expression of NFATc1 and its downstream genes, confirming the inhibitory effect of SPA0355 on RANKL-induced osteoclast differentiation.

In order to examine the in vivo effects of SPA0355 on bone remodeling, we used an OVX-induced osteoporosis model. The 10 and $50 \mathrm{mg} / \mathrm{kg}$ doses of SPA0355 used in this study are similar to those used in studies of rheumatoid arthritis [7] and streptozotocin-induced type 1 diabetes [8] and far greater than those used in studies of allergic airway inflammation [10] and hepatic ischemia-reperfusion injury [9]. It seems that doses of SPA0355 depend on the experimental period of the studies. OVX mice treated for five weeks with SPA0355 displayed improved trabecular femur BMD and other bone parameters (BV/TV, Tb.N, and Tb.Sp), which have been associated with bone loss after OVX. Unfortunately, no further improvement of any bone morphometric parameters measured by $\mu \mathrm{CT}$ scanning was observed with increase in SPA0355 dose from 10 to $50 \mathrm{mg} / \mathrm{kg}$. The lack of doseresponsiveness may be due to a dose-saturation effect. Alternatively, the mild nature of the parameter changes may suggest that a substantially larger dose than the five-fold difference we used may provide a dose-effect. However, in this case, unexpected toxic side effects may develop.

The limitations of this study should be considered. First, we only evaluated osteo-protective effects of SPA0355 at the end of the treatment period. We also did not optimize the specific dosage range. Second, although menopause has considered as the single most critical risk factor for osteoporosis, aging also has adverse effects on skeletal homeostasis independent of estrogen deficiency [18]. Therefore, further study should investigate the effects of SPA0355 on age-related changes in the bone itself. Finally, the direct interaction target of SPA0355 to inhibit the MAPKs, Akt, and NF- $\kappa$ B pathways has yet to be clarified.

In summary, we demonstrated that SPA0355 suppressed RANKL-induced osteoclastogenesis in BMMs by inhibiting the activation of MAPKs (p-38, ERK, and JNK), Akt, and NF- $\mathrm{B}$ and by suppressing the expression of NFATc1 (Fig. 7). This study discloses the beneficial effect of SPA0355 on OVX-induced bone resorption.

\section{ACKNOWLEDGEMENTS}

This research was supported by grants from the Medical Research Center Program (2011-0030074 and 2017R1A5A2015061) through the National Research Foundation of Korea (NRF), funded by the Korean government.

\section{CONFLICTS OF INTEREST}

The authors declare no conflicts of interest.

\section{REFERENCES}

1. Manolagas SC, Parfitt AM. What old means to bone. Trends Endocrinol Metab. 2010;21:369-374.

2. Fujiwara Y, Piemontese M, Liu Y, Thostenson JD, Xiong J, O’Brien 
CA. RANKL (Receptor Activator of $\mathrm{NF}_{\kappa} \mathrm{B}$ Ligand) produced by osteocytes is required for the increase in B cells and bone loss caused by estrogen deficiency in mice. J Biol Chem. 2016;291:24838-24850.

3. Darnay BG, Ni J, Moore PA, Aggarwal BB. Activation of NF-kappaB by RANK requires tumor necrosis factor receptor-associated factor (TRAF) 6 and NF-kappaB-inducing kinase. Identification of a novel TRAF6 interaction motif. J Biol Chem. 1999;274:7724-7731.

4. Eghbali-Fatourechi G, Khosla S, Sanyal A, Boyle WJ, Lacey DL, Riggs BL. Role of RANK ligand in mediating increased bone resorption in early postmenopausal women. J Clin Invest. 2003;111:12211230.

5. Matsumoto M, Sudo T, Saito T, Osada H, Tsujimoto M. Involvement of 38 mitogen-activated protein kinase signaling pathway in osteoclastogenesis mediated by receptor activator of NF-kappa B ligand (RANKL). J Biol Chem. 2000;275:31155-31161.

6. Boyle WJ, Simonet WS, Lacey DL. Osteoclast differentiation and activation. Nature. 2003;423:337-342.

7. Lee YR, Hwang JK, Lee HS, Cheon YJ, Ryu JH, Lee SI, Kwak HB, Lee SM, Kim JS, Park JW, Jeon R, Park BH. SPA0355, a thiourea analogue, inhibits inflammatory responses and joint destruction in fibroblast-like synoviocytes and mice with collagen-induced arthritis. Br J Pharmacol. 2011;164:794-806.

8. Bae UJ, Song MY, Jang HY, Gim HJ, Ryu JH, Lee SM, Jeon R, Park $\mathrm{BH}$. The efficacy of SPA0355 in protecting $\beta$ cells in isolated pancreatic islets and in a murine experimental model of type 1 diabetes. Exp Mol Med. 2013;45:e51.

9. Bae UJ, Yang JD, Ka SO, Koo JH, Woo SJ, Lee YR, Yu HC, Cho BH, Zhao HY, Ryu JH, Lee SM, Jeon R, Park BH. SPA0355 attenuates ischemia/reperfusion-induced liver injury in mice. Exp Mol Med. 2014;46:e109.
10. Jang HY, Jeon R, Kang KW, Song MY, Lim JM, Lee E, Ryu JH, Lee SM, Park BH. SPA0355 suppresses T-cell responses and reduces airway inflammation in mice. Eur J Pharmacol. 2014;745:19-28.

11. Lee Y, Ka SO, Cha HN, Chae YN, Kim MK, Park SY, Bae EJ, Park BH. Myeloid sirtuin 6 deficiency causes insulin resistance in highfat diet-fed mice by eliciting macrophage polarization toward an M1 phenotype. Diabetes. 2017;66:2659-2668.

12. Cao JJ, Gregoire BR, Sun L, Song S. Alpha-1 antitrypsin reduces ovariectomy-induced bone loss in mice. Ann N Y Acad Sci. 2011; 1240:E31-35.

13. Abuohashish HM, Ahmed MM, Al-Rejaie SS, Eltahir KE. The antidepressant bupropion exerts alleviating properties in an ovariectomized osteoporotic rat model. Acta Pharmacol Sin. 2015;36:209220.

14. Santen RJ, Kagan R, Altomare CJ, Komm B, Mirkin S, Taylor HS. Current and evolving approaches to individualizing estrogen receptor-based therapy for menopausal women. JClin Endocrinol Metab. 2014;99:733-747.

15. Zhao R. Immune regulation of osteoclast function in postmenopausal osteoporosis: a critical interdisciplinary perspective. Int $J$ Med Sci. 2012;9:825-832.

16. Rufus P, Mohamed N, Shuid AN. Beneficial effects of traditional Chinese medicine on the treatment of osteoporosis on ovariectomised rat models. Curr Drug Targets. 2013;14:1689-1693.

17. Wijkmans J, Gossen J. Inhibitors of cathepsin K: a patent review (2004 - 2010). Expert Opin Ther Pat. 2011;21:1611-1629.

18. Manolagas SC. From estrogen-centric to aging and oxidative stress: a revised perspective of the pathogenesis of osteoporosis. Endocr Rev. 2010;31:266-300. 